\title{
Depression among people with type 2 diabetes mellitus, US National Health and Nutrition Examination Survey (NHANES), 2005-2012
}

Yiting Wang ${ }^{1 *}$, Janice M. S. Lopez ${ }^{2}$, Susan C. Bolge ${ }^{2}$, Vivienne J. Zhu ${ }^{3}$ and Paul E. Stang ${ }^{1}$

\begin{abstract}
Background: Depression in people with diabetes can result in increased risk for diabetes-related complications. The prevalence of depression has been estimated to be $17.6 \%$ in people with type 2 diabetes mellitus (T2DM), based on studies published between 1980 and 2005. There is a lack of more recent estimates of depression prevalence among the US general T2DM population.
\end{abstract}

Methods: The present study used the US National Health and Nutrition Examination Survey (NHANES) 2005-2012 data to provide an updated, population-based estimate for the prevalence of depression in people with T2DM. NHANES is a cross-sectional survey of a nationally representative sample of the civilian, non-institutionalized US population. Starting from 2005, the Patient Health Questionnaire (PHQ-9) was included to measure signs and symptoms of depression. We defined PHQ-9 total scores $\geq 10$ as clinically relevant depression (CRD), and $\geq 15$ as clinically significant depression (CSD). Self-reported current antidepressant use was also combined to estimate overall burden of depression. Predictors of CRD and CSD were investigated using survey logistic regression models.

Results: A total of 2182 participants with T2DM were identified. The overall prevalence of CRD and CSD among people with T2DM is $10.6 \%$ (95\% confidence interval (Cl) 8.9-12.2\%), and $4.2 \%$ (95 \% Cl 3.4-5.1\%), respectively. The combined burden of depressive symptoms and antidepressants may be as high as $25.4 \%$ (95 \% Cl 23.0-27.9\%). Significant predictors of CRD include age (younger than 65), sex (women), income (lower than $130 \%$ of poverty level), education (below college), smoking (current or former smoker), body mass index ( $\geq 30 \mathrm{~kg} / \mathrm{m}^{2}$ ), sleep problems, hospitalization in the past year, and total cholesterol ( $\geq 200 \mathrm{mg} / \mathrm{dl})$. Significant predictors of CSD also include physical activity (below guideline) and cardiovascular diseases.

Conclusions: The prevalence of CRD and CSD among people with T2DM in the US may be lower than in earlier studies, however, the burden of depression remains high. Further research with longitudinal follow-up for depression in people with T2DM is needed to understand real world effectiveness of depression management.

Keywords: Depression, Patient Health Questionnaire (PHQ-9), Type 2 diabetes mellitus (T2DM)

\footnotetext{
* Correspondence: ywang28@its.jnj.com

${ }^{1}$ Janssen Research \& Development, LLC, 1125 Trenton Harbourton Road,

Titusville, NJ 08560, USA

Full list of author information is available at the end of the article
} 


\section{Background}

Depression can increase the risk of diabetes-related complications in people with diabetes due to poor self-care, reduced treatment adherence, and poor glycemic control [1]. The management of comorbid depression and diabetes through integrated and collaborative care has been shown to improve medication adherence, glycemic control [2], and depression outcomes [3]. Routine screening of depression in adults with diabetes is recommended by the American Association of Clinical Endocrinologists (AACE) [4]. Integrating depression screening with cost-effective treatment strategies has also been recommended for the general primary care setting [5]. Yet, it has been suggested that few diabetes clinics provide mental health screening or integrate mental/behavioral health services in diabetes clinical care, and even the use of brief paperand-pencil self-report measures remains rare [6].

There is extensive evidence that depression is a common comorbidity in people with diabetes [7]. One metaanalysis reported an overall depression prevalence of $27 \%$ in people with type 2 diabetes mellitus (T2DM) based on 15 studies published before January 2000 [8]. A later meta-analysis reported an overall depression prevalence of $17.6 \%$ in people with T2DM compared with $9.8 \%$ in those without T2DM, based on 10 studies published between January 1980 and May 2005 with a total of 51,331 people across multiple countries, including the US [9]. There is only a limited number of large, population-based studies that estimated depression prevalence for people with T2DM. The Kaiser Permanente Diabetes Registry reported a prevalence of diagnosed depression (based on diagnosis codes in medical records) to be $17.9 \%$ in T2DM in 1999 [10]. A study of the 2006 Preventive Behavioral Risk Factor Surveillance System (PBRFSS) (a cross-sectional, random-sample, telephone survey) reported depression prevalence of $24 \%$ in people with T2DM using insulin, and $17.3 \%$ in people with T2DM not using insulin (depression was defined as total score $\geq 10$ on the Patient Health Questionnaire, PHQ-8, administered in 36 states and territories in 2006) [11]. There is a lack of more recent, US national-level estimates of depression prevalence among a representative sample of the T2DM population.

The present study used the US National Health and Nutrition Examination Survey (NHANES) 2005-2012 data to estimate the prevalence of depression, and to explore potential predictors of clinically relevant and clinically significant depression (defined below) in a nationally representative sample that includes people with T2DM.

\section{Methods}

The US National Health and Nutrition Examination Survey (NHANES) is a cross-sectional survey of a nationally representative sample of the civilian, non-institutionalized US population, designed to monitor the nation's health and nutrition status [12]. NHANES uses a complex, multistage, probability sampling design to select participants, and continuous data collection has been conducted in 2-year cycles since 1999 [12]. During each 2-year cycle, about 30 selected counties (i.e., the primary sampling units) were visited out of approximately 3,000 counties in the US. Within selected segments of these counties, letters explaining NHANES were sent to selected households before trained interviewers visited and obtained informed consent from individuals for participation. Each 2-year survey cycle has a combined sample of roughly 10,000 persons and is representative of the civilian, non-institutionalized US population. Overall interview response rate was roughly $80 \%$ (see Additional file 1).

The survey comprises interviews conducted in participants' homes and standardized physical examinations in mobile examination centers (MECs), including laboratory tests. Topics covered by the survey questionnaires include demographics (e.g., age, sex, race/ethnicity, family income-to-poverty ratio [13], education, and marital status), general health (e.g., hospitalization in the past year, weight change history, hours of sleep, sleep problems), behavior and lifestyle variables (e.g., smoking, alcohol, physical activities, substance use), disease history (e.g., whether they have been diagnosed by health professionals to have diabetes, hypertension, cancer, cardiovascular diseases, liver disease). NHANES participants reported prescription medication use during a one-month period prior to the survey date, and were asked to show the interviewer their medication containers during the household interview. Lexicon Plus, a proprietary database of Cerner Multum, Inc. [14] was used in NHANES to assist with medication data collection, data editing and release. The Lexicon Plus ${ }^{\circledast}$ is a comprehensive database of all prescription and some nonprescription drug products available in the US drug market. We identified antidepressants using the Lexicon Plus ${ }^{\oplus}$ therapeutic classification (first-level category "PSYCHOTHERAPEUTIC AGENTS", secondlevel category "ANTIDEPRESSANTS"), which included selective serotonin reuptake inhibitors [SSRI], monoamine oxidase inhibitors [MAOI], tricyclic antidepressants [TCA], serotonin-norepinephrine reuptake inhibitors [SNRI], phenylpiperazine and miscellaneous antidepressants [15]. About $85 \%$ of the self-reported use of antidepressants had a record of "prescription container seen by the interviewer".

Starting from 2005, NHANES has included the Patient Health Questionnaire (PHQ-9) $[16,17]$ to measure signs and symptoms of depression. The PHQ-9 and some other computer-assisted personal interviews, and anthropometrics (e.g., body weight, height, waist-to-height ratio) as well as bio-specimens are taken in the MEC according to standardized protocols. The PHQ-9 is a 9- 
item depression screening instrument that asks about the frequency of symptoms of depression over the past 2 weeks $[16,17]$. Total PHQ-9 score ranges from 0 to 27 and are categorized as "none or minimum" (0-4), "mild" (5-9), "moderate" (10-14), "moderately severe" (15-19), and "severe" (20-27) for depression severity. In this study, we combined scores 10-27 ("moderately severe or severe") to improve precision of estimates. We defined PHQ-9 total scores $\geq 10$ as clinically relevant depression (CRD) [18], which is consistent with depression process/ outcome performance measures recommended by the National Quality Forum [19]. PHQ-9 score $\geq 10$ has shown a sensitivity of $88 \%$ and a specificity of $88 \%$ for major depression [17]. In addition, those with PHQ- 9 scores $\geq 15$ (a subset of those with PHQ-9 scores $\geq 10$ ) were categorized as clinically significant depression (CSD), which suggests the presence of major depression for which active treatment with pharmacotherapy and/or psychotherapy is recommended [17]. Participants reporting current antidepressant use were also included as prevalent cases in the estimation of overall burden of depressive symptoms because this may imply CRD and/or CSD. Because some antidepressants are also indicated for other psychiatric symptoms (e.g., anxiety), and because the reasons for taking antidepressants were not available from NHANES, we conducted sensitivity analyses of various combinations of PHQ-9 score, antidepressant use and consultation with mental health professionals. We also analyzed question 9 of the PHQ-9 questionnaire which asks about suicidal ideation. The specificity of the PHQ-9 suicide screening item was 0.84 and sensitivity was 0.69 in a study of 166 people from 2 primary care clinics in the US, which suggested it may be useful in primary care practice to identify individuals at risk for suicide [20]. In addition to the PHQ-9 questions, NHANES also assesses the difficulty caused by the depressive symptoms with work, taking care of things at home, or getting along with people, using a scale of 'not at all' to extremely difficult.

We identified people with T2DM using the following criteria [21, 22]: self-reported diagnosis of diabetes or "sugar diabetes" at age $\geq 30$ years, not initiating insulin therapy within 1 year of diabetes diagnosis, and not pregnant at the time of interview/examination. Diabetes-related variables such as age at diagnosis, self-monitoring of blood glucose, diabetes complications and antihyperglycemic medications were based on NHANES diabetes questionnaire data, lab/ exam variables such as glycohemoglobin (HbA1c), cholesterol, and blood pressure were based on NHANES MEC data.

Analyses were weighted, and accounted for the stratified, multistage probability sampling design of NHANES and survey nonresponse. Logistic regression models were used to select predictors of CRD and CSD in T2DM from potential candidate variables $[9,23]$, including demographics, general health, behavior and lifestyle, disease history, lab and prescription medications. First, univariable association of all the potential predictors were evaluated (i.e., entered into the survey logistic regression models one at a time), and those with statistically significant association with CRD and/or CSD were then entered together into a multivariable regression model. The final multivariable model retained all variables that remained statistically significant. Statistical significance was assessed by two-sided $P$ values of $<0.05$, with no adjustments for multiple testing. Odds ratio (OR) and $95 \%$ confidence intervals $(\mathrm{CI})$ were estimated and presented for predictors selected in the final models. We did not use stepwise automated model selection because the effective degrees of freedom are bounded by the number of clusters (i.e., primary sampling units in NHANES), which makes such methods problematic [24-26]. We also compared analyses that excluded missing PHQ-9 data with analyses that combined missing PHQ-9 data with the non-CRD and nonCSD categories.

We also evaluated whether there was a temporal trend in the prevalence of depressive symptoms or antidepressant use over the 8 years (i.e., 4 NHANES survey cycles). This was done by fitting NHANES survey cycle as a continuous variable in the survey regression models. All analyses were performed using SAS (SAS Institute Inc., Cary, NC, USA), version 9.2.

\section{Results}

There were a total of 40,790 participants in the NHANES 2005-2012 (which represents the total US civilian, noninstitutionalized population of 299.3 million people). Among these, a total of 2,648 respondents reported that they had been "told by a doctor or health professional" that they had "diabetes or sugar diabetes" (this sample represents 18.8 million people with diagnosed diabetes). From the 2,648 respondents, we identified 2,182 participants with T2DM by our definition and who had data for both the interview and physical examination. Among the 2,182 respondents with T2DM, 235 (10.8 \%) had missing data on one or more items of the PHQ-9 questionnaire, including 37 (1.7\%) taking antidepressants. We compared analyses that excluded missing PHQ-9 data with analyses that combined the missing data with the non-CRD and non-CSD categories. Results were similar and the latter are presented.

Tables 1 and 2 describe the general characteristics of people with T2DM according to clinically relevant depression (CRD, defined by PHQ- 9 total score $>=10$ ) and clinically significant depression (CSD, defined by PHQ-9 total score $>=15$ ). (See Additional file 2 for the descriptions according to the four PHQ-9 categories). Overall, the categories for more severe depression have a higher proportion of T2DM subjects who were younger, women, minority 
Table 1 Characteristics of people with T2DM by clinically relevant depression, NHANES 2005-2012

\begin{tabular}{|c|c|c|c|}
\hline & Non-CRD & CRD & Overall \\
\hline N, participants (\%) & $1926(88.3)$ & $256(11.7)$ & $2182(100.0)$ \\
\hline Frequency, weighted ${ }^{\mathrm{a}}(\%)$ & $13,520,141(89.4)$ & $1,597,475(10.6)$ & $15,117,616(100.0)$ \\
\hline \multicolumn{4}{|l|}{ Age group, \% } \\
\hline 30-49 years & 15.4 & 27.5 & 16.6 \\
\hline $50-64$ years & 38.1 & 43.7 & 38.7 \\
\hline $65-74$ years & 26.7 & 18.8 & 25.8 \\
\hline$\geq 75$ years & 19.9 & 10.0 & 18.8 \\
\hline \multicolumn{4}{|l|}{ Sex $\%$} \\
\hline Male & 50.8 & 30.7 & 48.7 \\
\hline Female & 49.2 & 69.3 & 51.3 \\
\hline \multicolumn{4}{|l|}{ Race, \% } \\
\hline Non-Hispanic white & 61.1 & 54.6 & 60.4 \\
\hline Non-Hispanic black & 17.3 & 21.6 & 17.8 \\
\hline Mexican and other Hispanic & 12.9 & 19.9 & 13.6 \\
\hline Other & 8.7 & 3.9 & 8.2 \\
\hline Ratio of family income to poverty level $\leq 1.3, \%$ & 20.8 & 52.1 & 24.1 \\
\hline Marital status, single or living alone, \% & 36.3 & 54.5 & 38.3 \\
\hline \multicolumn{4}{|l|}{ Education, \% } \\
\hline College or above & 47.2 & 31.6 & 45.5 \\
\hline High school graduate & 25.3 & 22.6 & 25.0 \\
\hline Below high school & 27.5 & 45.8 & 29.4 \\
\hline \multicolumn{4}{|l|}{ Smoking } \\
\hline Non-smoker & 51.0 & 39.3 & 49.8 \\
\hline Past & 36.0 & 28.5 & 35.2 \\
\hline Current & 13.0 & 32.2 & 15.0 \\
\hline \multicolumn{4}{|l|}{ Alcohol } \\
\hline Non-drinker & 42.4 & 49.4 & 43.2 \\
\hline Above moderate level & 16.0 & 21.2 & 16.6 \\
\hline Moderate drinking & 41.5 & 29.4 & 40.2 \\
\hline Marijuana, ever use, \% & 16.5 & 28.9 & 17.8 \\
\hline Physical activity level met guidelines, ${ }^{b} \%$ & 43.1 & 26.7 & 41.4 \\
\hline Cardiovascular diseases & 28.0 & 36.4 & 28.8 \\
\hline Diabetic retinopathy & 18.0 & 25.4 & 18.8 \\
\hline Hypertension & 81.7 & 81.5 & 81.7 \\
\hline Liver diseases & 4.1 & 7.7 & 4.5 \\
\hline Cancer & 17.4 & 18.5 & 17.5 \\
\hline Weak/failing kidneys ${ }^{c}$ & 7.3 & 12.2 & 7.8 \\
\hline Overnight hospitalization in the past year, \% & 20.5 & 44.0 & 23.0 \\
\hline \multicolumn{4}{|l|}{ My health in general is, \% } \\
\hline Excellent, very good & 16.3 & 2.5 & 14.8 \\
\hline Good/Fair & 69.6 & 65.2 & 69.1 \\
\hline Poor & 6.5 & 32.4 & 9.2 \\
\hline Missing & 7.7 & 0.0 & 6.9 \\
\hline
\end{tabular}


Table 1 Characteristics of people with T2DM by clinically relevant depression, NHANES 2005-2012 (Continued)

\begin{tabular}{|c|c|c|c|}
\hline \multicolumn{4}{|l|}{ Hours of sleep per day, $\%$} \\
\hline$<=5 \mathrm{~h}$ & 16.2 & 38.5 & 18.6 \\
\hline $6-8 \mathrm{~h}$ & 74.1 & 50.7 & 71.6 \\
\hline$>=9 \mathrm{~h}$ & 9.1 & 9.9 & 9.2 \\
\hline Missing & 0.5 & 1.0 & 0.6 \\
\hline Self-reported having trouble sleep, \% & 33.8 & 65.2 & 37.1 \\
\hline Told by doctor having sleep disorders, $\%$ & 15.7 & 29.9 & 17.2 \\
\hline \multicolumn{4}{|l|}{ Age at diabetes diagnosis, years } \\
\hline Mean (s.e.) & $52.6(0.4)$ & $48.9(0.9)$ & $52.2(0.4)$ \\
\hline \multicolumn{4}{|l|}{ Categories, \% } \\
\hline$<50$ & 41.6 & 55.6 & 43.0 \\
\hline $50-69$ & 47.9 & 38.3 & 46.8 \\
\hline$>=70$ & 9.8 & 4.8 & 9.3 \\
\hline Missing & 0.8 & 1.3 & 0.8 \\
\hline \multicolumn{4}{|l|}{ Duration of diabetes } \\
\hline Mean (s.e.) & $10.0(0.2)$ & $8.9(0.7)$ & $9.9(0.2)$ \\
\hline \multicolumn{4}{|l|}{ Categories, \% } \\
\hline$<5$ years & 30.8 & 28.3 & 30.5 \\
\hline $5-9$ years & 23.9 & 29.9 & 24.5 \\
\hline$>=10$ years & 44.5 & 40.6 & 44.1 \\
\hline Missing & 0.8 & 1.3 & 0.8 \\
\hline \multicolumn{4}{|l|}{$\mathrm{HbA} 1 \mathrm{c}, \%$} \\
\hline Mean (s.e.) & $7.3(0.1)$ & $7.4(0.2)$ & $7.3(0.1)$ \\
\hline \multicolumn{4}{|l|}{ Categories, \% } \\
\hline$<6.5 \%$ & 34.8 & 34.0 & 34.7 \\
\hline $6.5-<7.5 \%$ & 29.1 & 25.3 & 28.7 \\
\hline $7.5-8.9 \%$ & 18.9 & 17.9 & 18.8 \\
\hline$>=9 \%$ & 13.0 & 13.4 & 13.0 \\
\hline Missing & 4.2 & 9.5 & 4.7 \\
\hline \multicolumn{4}{|l|}{ Total cholesterol (mg/dl) } \\
\hline Mean (s.e.) & $182.0(1.6)$ & $193.1(3.7)$ & $183.1(1.5)$ \\
\hline \multicolumn{4}{|l|}{ Categories } \\
\hline$>0$ and $<200$ & 65.8 & 49.8 & 64.1 \\
\hline$>=200$ & 27.9 & 39.9 & 29.2 \\
\hline Missing & 6.3 & 10.3 & 6.7 \\
\hline \multicolumn{4}{|l|}{ LDL cholesterol(mg/dl) } \\
\hline Mean (s.e.) & $98.1(1.4)$ & $108.4(4.1)$ & $99.0(1.4)$ \\
\hline \multicolumn{4}{|l|}{ Categories } \\
\hline$>0$ and $<100$ & 52.7 & 44.9 & 52.0 \\
\hline$>=100$ & 39.4 & 48.5 & 40.2 \\
\hline Missing & 7.9 & 6.6 & 7.8 \\
\hline \multicolumn{4}{|l|}{ HDL cholesterol (mg/dl) } \\
\hline Male and female, mean (s.e.) & $47.8(0.4)$ & $45.8(1.1)$ & $47.6(0.4)$ \\
\hline Male, mean (s.e.) & $44.2(0.5)$ & $41.2(2.0)$ & $44.0(0.5)$ \\
\hline Female, mean (s.e.) & $51.7(0.7)$ & $48.0(1.2)$ & $51.2(0.7)$ \\
\hline
\end{tabular}


Table 1 Characteristics of people with T2DM by clinically relevant depression, NHANES 2005-2012 (Continued)

\begin{tabular}{|c|c|c|c|}
\hline \multicolumn{4}{|l|}{ Categories, \% } \\
\hline Male $>40$ and female $>50$ & 49.7 & 36.9 & 48.4 \\
\hline Male $<=40$ and female $<=50$ & 43.9 & 52.8 & 44.9 \\
\hline Missing & 6.3 & 10.3 & 6.7 \\
\hline \multicolumn{4}{|l|}{ Triglycerides (mg/dl) } \\
\hline Mean (s.e.) & $176.3(9.8)$ & $180.1(14.0)$ & $176.6(9.4)$ \\
\hline \multicolumn{4}{|l|}{ Categories } \\
\hline$>0$ and $<150$ & 55.6 & 46.3 & 54.8 \\
\hline$>=150$ & 41.8 & 50.4 & 42.6 \\
\hline Missing & 2.5 & 3.3 & 2.6 \\
\hline \multicolumn{4}{|l|}{ Blood pressure (BP), mmHg } \\
\hline SBP, mean (s.e.) & $130.6(0.6)$ & $128.6(1.4)$ & $130.4(0.5)$ \\
\hline DBP, mean (s.e) & $68.4(0.5)$ & $70.7(1.0)$ & $68.6(0.4)$ \\
\hline $\mathrm{SBP}<140, \mathrm{DBP}<90$ & 67.1 & 72.3 & 67.5 \\
\hline Missing & 4.9 & 2.3 & 4.6 \\
\hline \multicolumn{4}{|l|}{ Medications } \\
\hline Insulin, \% & 27.0 & 24.7 & 26.8 \\
\hline Sulfonylureas, \% & 35.8 & 27.0 & 34.8 \\
\hline THIAZOLIDINEDIONES, \% & 17.5 & 14.3 & 17.1 \\
\hline Meglitinides, \% & 2.0 & 0.2 & 1.8 \\
\hline DPP-4 INHIBITORS, \% & 7.6 & 1.4 & 6.9 \\
\hline GLP-1 AGONISTS, \% & 1.5 & 1.8 & 1.5 \\
\hline Metformin, \% & 52.2 & 59.7 & 53.0 \\
\hline Any antihyperglycemic agents & 88.1 & 87.3 & 88.0 \\
\hline Antihypertensives, \% & 76.7 & 76.0 & 76.6 \\
\hline Statins, \% & 54.3 & 52.0 & 54.1 \\
\hline \multicolumn{4}{|l|}{ Body mass index, $\mathrm{kg} / \mathrm{m}^{2}$} \\
\hline Mean (s.e.) & $32.5(0.3)$ & $35.5(0.6)$ & $32.8(0.2)$ \\
\hline \multicolumn{4}{|l|}{ Categories, \% } \\
\hline$<25$ & 13.9 & 8.2 & 13.3 \\
\hline $25-<30$ & 26.7 & 11.0 & 25.0 \\
\hline $30-<35$ & 27.4 & 37.1 & 28.5 \\
\hline $35+$ & 31.9 & 43.7 & 33.2 \\
\hline Missing & 0.1 & 0.0 & 0.1 \\
\hline Waist-to-height ratio, mean (s.e.) & $0.659(0.004)$ & $0.699(0.007)$ & $0.663(0.003)$ \\
\hline \multicolumn{4}{|l|}{ Categories, \% } \\
\hline $0.3-<0.5$ & 2.4 & 1.4 & 2.3 \\
\hline $0.5-<0.7$ & 62.6 & 48.4 & 61.1 \\
\hline$\geq 0.7$ & 26.8 & 40.2 & 28.2 \\
\hline Missing & 8.3 & 10.0 & 8.5 \\
\hline
\end{tabular}

Values are \% unless stated otherwise, percentages may not add up exactly to 100 due to rounding

CRD clinically relevant depression, defined by PHQ-9 score $\geq 10$; S.e. $=$ standard error

${ }^{a}$ There were 466 participants $(=2,648-2,182)$ who reported having diagnosed diabetes but were not included in this analysis (due to presumed type 1 diabetes or non-participation in the MEC exams), representing 3,653,851 $(=18,771,467-15,117,616)$ people with diabetes in the US civilian, non-institutionalized population b Physical activity level evaluated according to the 2008 Physical Activity Guidelines for Americans [36]

'A "yes" answer to "Have you ever been told by a doctor or other health professional that you have weak or failing kidneys (excluding kidney stones, bladder infections, or incontinence)?" 
Table 2 Characteristics of people with T2DM by clinically significant depression, NHANES 2005-2012

\begin{tabular}{|c|c|c|c|}
\hline & Non-CSD & CSD & Overall \\
\hline N, participants & $1926(88.3)$ & $256(11.7)$ & 2182 \\
\hline Frequency, weighted ${ }^{\mathrm{a}}(\%)$ & $13,520,141(89.4)$ & $1,597,475(10.6)$ & $15,117,616(100.0)$ \\
\hline \multicolumn{4}{|l|}{ Age group, \% } \\
\hline 30-49 years & 16.3 & 25.4 & 16.6 \\
\hline $50-64$ years & 38.2 & 50.3 & 38.7 \\
\hline $65-74$ years & 26.3 & 15.4 & 25.8 \\
\hline$\geq 75$ years & 19.3 & 8.9 & 18.8 \\
\hline \multicolumn{4}{|l|}{ Sex $\%$} \\
\hline Male & 49.5 & 29.4 & 48.7 \\
\hline Female & 50.5 & 70.6 & 51.3 \\
\hline \multicolumn{4}{|l|}{ Race, \% } \\
\hline Non-Hispanic white & 60.8 & 50.6 & 60.4 \\
\hline Non-Hispanic black & 17.6 & 23.4 & 17.8 \\
\hline Mexican and other Hispanic & 13.3 & 21.8 & 13.6 \\
\hline Other & 8.3 & 4.2 & 8.2 \\
\hline Ratio of family income to poverty level $\leq 1.3, \%$ & 22.4 & 62.9 & 24.1 \\
\hline Marital status, single or living alone, \% & 37.6 & 53.3 & 38.3 \\
\hline \multicolumn{4}{|l|}{ Education, \% } \\
\hline College or above & 46.5 & 23.7 & 45.5 \\
\hline High school graduate & 25.3 & 19.5 & 25.0 \\
\hline Below high school & 28.2 & 56.8 & 29.4 \\
\hline \multicolumn{4}{|l|}{ Smoking } \\
\hline Non-smoker & 50.2 & 39.1 & 49.8 \\
\hline Past & 35.7 & 24.7 & 35.2 \\
\hline Current & 14.1 & 36.1 & 15.0 \\
\hline \multicolumn{4}{|l|}{ Alcohol } \\
\hline Non-drinker & 42.5 & 58.9 & 43.2 \\
\hline Above moderate level & 16.4 & 20.3 & 16.6 \\
\hline Moderate drinking & 41.1 & 20.8 & 40.2 \\
\hline Marijuana, ever use, \% & 17.3 & 29.3 & 17.8 \\
\hline Physical activity level met guidelines, ${ }^{b} \%$ & 42.5 & 16.9 & 41.4 \\
\hline Cardiovascular diseases & 28.3 & 41.9 & 28.8 \\
\hline Diabetic retinopathy & 18.8 & 18.6 & 18.8 \\
\hline Hypertension & 81.8 & 79.1 & 81.7 \\
\hline Liver diseases & 4.2 & 11.9 & 4.5 \\
\hline Cancer & 17.3 & 23.8 & 17.5 \\
\hline Weak/failing kidneys ${ }^{c}$ & 7.6 & 13.1 & 7.8 \\
\hline Overnight hospitalization in the past year, \% & 22.1 & 42.5 & 23.0 \\
\hline \multicolumn{4}{|l|}{ My health in general is, \% } \\
\hline Excellent, very good & 15.4 & 1.2 & 14.8 \\
\hline Good/Fair & 69.4 & 61.7 & 69.1 \\
\hline Poor & 8.0 & 37.1 & 9.2 \\
\hline Missing & 7.2 & 0.0 & 6.9 \\
\hline
\end{tabular}


Table 2 Characteristics of people with T2DM by clinically significant depression, NHANES 2005-2012 (Continued)

\begin{tabular}{|c|c|c|c|}
\hline \multicolumn{4}{|l|}{ Hours of sleep per day, $\%$} \\
\hline$<=5 \mathrm{~h}$ & 17.5 & 43.5 & 18.6 \\
\hline $6-8 \mathrm{~h}$ & 73.0 & 40.2 & 71.6 \\
\hline$>=9 \mathrm{~h}$ & 8.9 & 15.6 & 9.2 \\
\hline Missing & 0.6 & 0.7 & 0.6 \\
\hline Self-reported having trouble sleep, \% & 35.7 & 69.2 & 37.1 \\
\hline Told by doctor having sleep disorders, $\%$ & 16.3 & 38.2 & 17.2 \\
\hline \multicolumn{4}{|l|}{ Age at diabetes diagnosis, years } \\
\hline Mean (s.e.) & $52.4(0.4)$ & $48.4(1.3)$ & $52.2(0.4)$ \\
\hline \multicolumn{4}{|l|}{ Categories, \% } \\
\hline$<50$ & 42.5 & 56.1 & 43.0 \\
\hline $50-69$ & 47.3 & 37.2 & 46.8 \\
\hline$>=70$ & 9.5 & 4.8 & 9.3 \\
\hline Missing & 0.8 & 1.9 & 0.8 \\
\hline \multicolumn{4}{|l|}{ Duration of diabetes } \\
\hline Mean (s.e.) & $9.9(0.2)$ & $9.6(1.0)$ & $9.9(0.2)$ \\
\hline \multicolumn{4}{|l|}{ Categories, \% } \\
\hline$<5$ years & 30.9 & 21.1 & 30.5 \\
\hline $5-9$ years & 24.3 & 29.3 & 24.5 \\
\hline$>=10$ years & 43.9 & 47.6 & 44.1 \\
\hline Missing & 0.8 & 1.9 & 0.8 \\
\hline \multicolumn{4}{|l|}{$\mathrm{HbA} 1 \mathrm{c}, \%$} \\
\hline Mean (s.e.) & $7.3(0.1)$ & $7.4(0.2)$ & $7.3(0.1)$ \\
\hline \multicolumn{4}{|l|}{ Categories, \% } \\
\hline$<6.5 \%$ & 34.9 & 31.7 & 34.7 \\
\hline $6.5-<7.5 \%$ & 28.7 & 28.7 & 28.7 \\
\hline $7.5-8.9 \%$ & 19.1 & 11.9 & 18.8 \\
\hline$>=9 \%$ & 12.9 & 15.0 & 13.0 \\
\hline Missing & 4.4 & 12.8 & 4.7 \\
\hline \multicolumn{4}{|l|}{ Total cholesterol (mg/dl) } \\
\hline Mean (s.e.) & $182.6(1.6)$ & $195.3(5.3)$ & $183.1(1.5)$ \\
\hline \multicolumn{4}{|l|}{ Categories } \\
\hline$>0$ and $<200$ & 65.1 & 42.4 & 64.1 \\
\hline$>=200$ & 28.5 & 43.4 & 29.2 \\
\hline Missing & 6.4 & 14.2 & 6.7 \\
\hline \multicolumn{4}{|l|}{ LDL cholesterol(mg/dl) } \\
\hline Mean (s.e.) & $98.5(1.4)$ & $112.9(6.0)$ & $99.0(1.4)$ \\
\hline \multicolumn{4}{|l|}{ Categories } \\
\hline$>0$ and $<100$ & 52.5 & 38.9 & 52.0 \\
\hline$>=100$ & 39.7 & 54.3 & 40.2 \\
\hline Missing & 7.8 & 6.8 & 7.8 \\
\hline \multicolumn{4}{|l|}{ HDL cholesterol (mg/dl) } \\
\hline Male and female, mean (s.e.) & $47.7(0.4)$ & $45.1(1.7)$ & $47.6(0.4)$ \\
\hline Male, mean (s.e.) & $44.1(0.5)$ & $38.7(2.7)$ & $44.0(0.5)$ \\
\hline Female, mean (s.e.) & $51.3(0.7)$ & $48.0(1.6)$ & $51.2(0.7)$ \\
\hline
\end{tabular}


Table 2 Characteristics of people with T2DM by clinically significant depression, NHANES 2005-2012 (Continued)

\begin{tabular}{|c|c|c|c|}
\hline \multicolumn{4}{|l|}{ Categories, \% } \\
\hline Male $>40$ and female $>50$ & 49.1 & 31.7 & 48.4 \\
\hline Male $<=40$ and female $<=50$ & 44.5 & 54.0 & 44.9 \\
\hline Missing & 6.4 & 14.2 & 6.7 \\
\hline \multicolumn{4}{|l|}{ Triglycerides (mg/dl) } \\
\hline Mean (s.e.) & $177.1(9.7)$ & $162.4(14.7)$ & $176.6(9.4)$ \\
\hline \multicolumn{4}{|l|}{ Categories } \\
\hline$>0$ and $<150$ & 55.1 & 47.6 & 54.8 \\
\hline$>=150$ & 42.5 & 45.5 & 42.6 \\
\hline Missing & 2.4 & 6.8 & 2.6 \\
\hline \multicolumn{4}{|l|}{ Blood pressure (BP), mmHg } \\
\hline SBP, mean (s.e.) & $130.5(0.6)$ & $126.6(2.1)$ & $130.4(0.5)$ \\
\hline DBP, mean (s.e) & $68.6(0.4)$ & $68.8(1.9)$ & $68.6(0.4)$ \\
\hline $\mathrm{SBP}<140, \mathrm{DBP}<90$ & 67.7 & 64.3 & 67.5 \\
\hline Missing & 4.6 & 4.5 & 4.6 \\
\hline \multicolumn{4}{|l|}{ Medications } \\
\hline Insulin, \% & 26.6 & 30.3 & 26.8 \\
\hline Sulfonylureas, \% & 35.4 & 22.1 & 34.8 \\
\hline THIAZOLIDINEDIONES, \% & 17.5 & 9.9 & 17.1 \\
\hline Meglitinides, \% & 1.8 & 0.4 & 1.8 \\
\hline DPP-4 INHIBITORS, \% & 7.3 & 0.0 & 6.9 \\
\hline GLP-1 AGONISTS, \% & 1.5 & 2.9 & 1.5 \\
\hline Metformin, \% & 53.0 & 53.7 & 53.0 \\
\hline Any antihyperglycemic agents & 88.3 & 81.6 & 88.0 \\
\hline Antihypertensives, \% & 76.8 & 73.2 & 76.6 \\
\hline Statins, \% & 54.5 & 45.2 & 54.1 \\
\hline \multicolumn{4}{|l|}{ Body mass index, kg/m² } \\
\hline Mean (s.e.) & $32.7(0.2)$ & $34.4(0.7)$ & $32.8(0.2)$ \\
\hline \multicolumn{4}{|l|}{ Categories, \% } \\
\hline$<25$ & 13.5 & 9.6 & 13.3 \\
\hline $25-<30$ & 25.5 & 13.5 & 25.0 \\
\hline $30-<35$ & 28.1 & 35.9 & 28.5 \\
\hline $35+$ & 32.8 & 41.0 & 33.2 \\
\hline Missing & 0.1 & 0.0 & 0.1 \\
\hline Waist-to-height ratio, mean (s.e.) & $0.662(0.003)$ & $0.691(0.010)$ & $0.663(0.003)$ \\
\hline \multicolumn{4}{|l|}{ Categories, \% } \\
\hline $0.3-<0.5$ & 2.3 & 1.8 & 2.3 \\
\hline $0.5-<0.7$ & 61.7 & 46.5 & 61.1 \\
\hline$\geq 0.7$ & 27.8 & 37.4 & 28.2 \\
\hline Missing & 8.2 & 14.3 & 8.5 \\
\hline
\end{tabular}

Values are \% unless stated otherwise, percentages may not add up exactly to 100 due to rounding. CSD Clinically significant depression, defined by PHQ-9 score $\geq 15$; S.e. $=$ standard error

${ }^{a}$ There were 466 participants $(=2,648-2,182)$ who reported having diagnosed diabetes but were not included in this analysis (due to presumed type 1 diabetes or non-participation in the MEC exams), representing 3,653,851 $(=18,771,467-15,117,616)$ people with diabetes in the US civilian, non-institutionalized population ${ }^{\mathrm{b}}$ Physical activity level evaluated according to the 2008 Physical Activity Guidelines for Americans [42]

"A "yes" answer to "Have you ever been told by a doctor or other health professional that you have weak or failing kidneys (excluding kidney stones, bladder infections, or incontinence)?" 
ethnicities (i.e., not non-Hispanic White), single or living alone, less physically active, having lower income, lower education level, sleep problems, cardiovascular diseases, cancer, liver and kidney diseases.

Figure 1 plots prevalence estimates of CRD and CSD. Between 2005 and 2012, the prevalence of CRD among people with T2DM is $10.6 \%$ overall (95 \% CI 8.9-12.2\%), and the prevalence is higher in women than in men. Similarly, the overall T2DM population prevalence of CSD is 4.2 \% (95 \% CI 3.4-5.1 \%). However, when antidepressant medication is taken into consideration, the combined burden of depression may be as high as $25.4 \%$ overall (95 \% CI 23.0-27.9\%). In a sensitivity analysis that removed some common antidepressants (see Table 3, last row) approved for other psychiatric indications (mainly anxiety and related disorders) in the US, the overall burden of CRD and/or antidepressants was $16.7 \%$ (95\% CI 14.6-18.9\%), and the overall burden of CSD and/or antidepressants was $10.8 \%$ (95 \% CI 8.9-12.7 \% in people with T2DM. In women, the corresponding prevalence were $20.5 \%$ (95 \% CI 16.7-24.3\%), and $12.3 \%$ (95 \% CI 9.2-15.4\%); in men, the corresponding prevalence were 12.7 \% (95 \% CI 10.5-15.0 \%) and $9.2 \%$ (95 \% CI 7.3$11.1 \%)$, respectively. There was a statistically significant trend in the prevalence of CRD and CSD, which increased early on and then stabilized from the 2009-2010 to the 2011-2012 period (Additional file 3). The increasing trend was noted at higher PHQ-9 scores (i.e., PHQ-9 score $\geq 15$ and PHQ-9 score $\geq 20$ ). No significant trend was detected in the prevalence of antidepressant use, although there appeared to be a drop in antidepressant use from the 2005-2006 to the 2007-2008 period. We did not find any notable differences in missing data pattern for PHQ-9 data across these years.

Table 3 characterized the general impact on health and life according to the severity of depressive symptoms based on PHQ-9 scores. Among people with T2DM who had moderately severe or severe depressive symptoms (PHQ-9 score $\geq 15), 23.2 \%$ reported that these problems made it extremely difficult for them to do their work, take care of things at home, or get along with people. In contrast, less than $1 \%$ of those with none or minimal depressive symptoms (PHQ-9 score 0-4) reported such negative impact. Further, about 1 in 5 of the subjects with moderately severe or severe depressive symptoms reported having suicidal thoughts nearly every day. Well over half of the people with moderately severe or severe depressive symptoms reported poor mental health and poor physical health over half of the past 30 days. With respect to combined impact of poor mental and poor physical health, $55.3 \%$ of people with moderately severe or severe depressive symptoms reported that poor physical or poor mental health kept them from usual activities such as self-care, work, school or recreation in more than half of the past 30 days. About half of the people with moderate or severe depressive symptoms reported taking antidepressants, but only 1 in 7 reported having seen or talked to a mental health professional such as a psychologist, psychiatrist, psychiatric nurse, or clinical social worker about their health during the past 12 months.

Figure 2 plots the mean PHQ-9 scores for statistically significant variables in the univariate analysis that were associated with CRD (i.e., PHQ-9 score $\geq 10$ ), and corresponding crude odds ratio (OR) estimates from the survey logistic regression models. Overall, the mean PHQ-9 total score in people with T2DM was 3.9 (95 \% CI 3.6-4.1). The OR estimates are consistent with descriptions in Table 1, for example, people with T2DM in the age category of 30-49 were almost 3-fold as likely to have CRD as those aged 65 years or older, with OR $=2.9(95 \% \mathrm{CI}$ 1.9-4.5). Female sex is associated with an OR of 2.3 (95\% CI 1.7-3.3) for CRD. Lower income (family income less than $130 \%$ of poverty level) is associated with an OR of 4.1 (95 \% CI 2.8-6.0) for CRD. Body mass index greater than 30 (i.e., obesity) or 35 (i.e., severe obesity) is associated with CRD with ORs about 2.

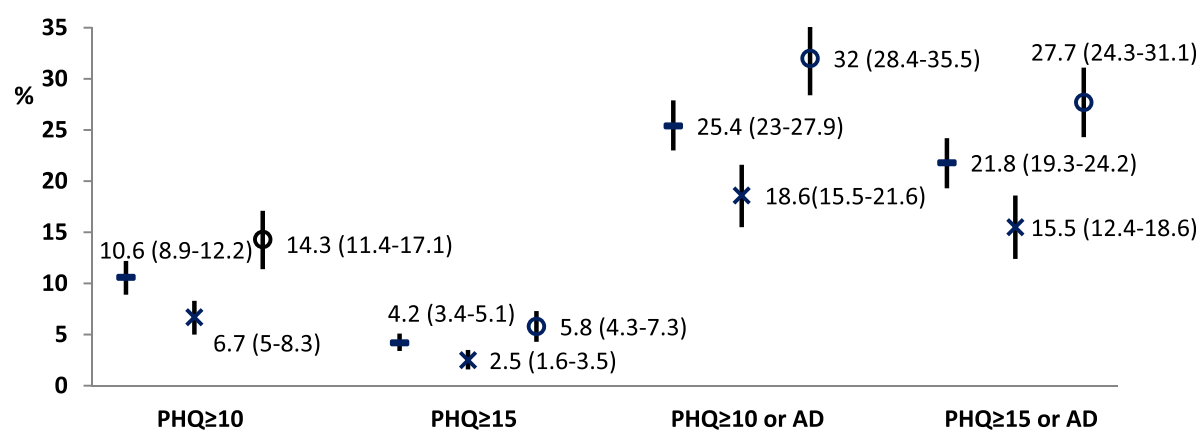

Fig. 1 Prevalence of CRD, CSD and antidepressant treatment in T2DM, NHANES 2005-2012. Legends: prevalence in men and women with T2DM overall shown by "-", in men with T2DM by " $x$ ", and in women with T2DM by "O", respectively. Vertical lines extend from lower to upper $95 \%$ confidence limits of the corresponding prevalence estimates. CRD, clinically relevant depression, defined by PHQ score $\geq 10 ;$ CSD, clinically significant depression, defined by $\mathrm{PHQ}$ score $\geq 15$; AD, currently taking antidepressants 
Table 3 Mental health status by severity of depressive symptoms

\begin{tabular}{|c|c|c|c|c|c|c|}
\hline \multirow[t]{3}{*}{ Characteristic } & \multicolumn{5}{|c|}{ PHQ-9 depression score } & \multirow[t]{3}{*}{ Overall } \\
\hline & \multirow{2}{*}{$\begin{array}{l}0-4 \\
\text { None/ } \\
\text { minimal }\end{array}$} & \multirow{2}{*}{$\begin{array}{l}\text { 5-9 } \\
\text { Mild }\end{array}$} & \multirow{2}{*}{$\begin{array}{l}\text { 10-14 } \\
\text { Moderate }\end{array}$} & $15-27$ & \multirow[t]{2}{*}{ Missing } & \\
\hline & & & & $\begin{array}{l}\text { Moderately } \\
\text { severe/Severe }\end{array}$ & & \\
\hline \multicolumn{7}{|c|}{ Difficulty with work, life, people due to depressive symptoms, \% } \\
\hline Not at all & 48.0 & 56.5 & 37.6 & 22.3 & 2.8 & 43.4 \\
\hline Somewhat & 9.5 & 39.6 & 42.1 & 22.2 & 1.4 & 16.5 \\
\hline Very & 0.8 & 2.8 & 17.0 & 31.6 & 0.4 & 3.4 \\
\hline Extreme & 0.2 & 1.1 & 3.3 & 23.2 & 0.5 & 1.5 \\
\hline Missing & 41.6 & 0.0 & 0.0 & 0.7 & 94.9 & 35.2 \\
\hline \multicolumn{7}{|l|}{ Suicidal ideation, \% } \\
\hline Not at all & 99.5 & 90.4 & 83.9 & 55.6 & 6.2 & 86.3 \\
\hline Several days & 0.5 & 8.9 & 14.4 & 13.9 & 0.7 & 3.4 \\
\hline More than half the days & 0 & 0.3 & 0.8 & 11.1 & 0.2 & 0.6 \\
\hline Nearly every day & 0 & 0.4 & 0.9 & 19.4 & 0 & 0.9 \\
\hline Missing & 0 & 0 & 0 & 0 & 92.9 & 8.8 \\
\hline \multicolumn{7}{|c|}{ \# of days mental health not good during the past 30 days } \\
\hline Mean (s.e.) & $2.0(0.2)$ & $6.9(0.6)$ & $13.4(1.3)$ & $19.5(1.3)$ & $5.4(1.4)$ & $4.6(0.3)$ \\
\hline \multicolumn{7}{|l|}{ Categories, \% } \\
\hline$<15$ days & 94.4 & 80.5 & 53.6 & 30.5 & 21.4 & 79.9 \\
\hline$>=15$ and $<=30$ & 5.5 & 19.4 & 46.4 & 68.2 & 4.0 & 13.0 \\
\hline Missing & 0.1 & 0.1 & 0.0 & 1.3 & 74.6 & 7.2 \\
\hline \multicolumn{7}{|c|}{ \# of days physical health not good during the past 30 days } \\
\hline Mean (s.e.) & $3.9(0.3)$ & $8.7(0.8)$ & $16.1(1.3)$ & $18.9(1.3)$ & $6.3(1.4)$ & $6.4(0.3)$ \\
\hline \multicolumn{7}{|l|}{ Categories, \% } \\
\hline$<15$ days & 89.0 & 73.9 & 46.8 & 36.6 & 20.8 & 75.1 \\
\hline$>=15$ and $<=30$ & 10.8 & 26.1 & 53.2 & 62.9 & 5.4 & 17.8 \\
\hline Missing & 0.2 & 0.1 & 0.0 & 0.5 & 73.8 & 7.1 \\
\hline \multicolumn{7}{|c|}{$\begin{array}{l}\text { \# of days during the past } 30 \text { days, poor physical/mental health } \\
\text { kept from doing usual activities, such as self-care, work, school } \\
\text { or recreation }\end{array}$} \\
\hline Mean (set 77, $99=)$. & $1.8(0.2)$ & $5.2(0.7)$ & $11.8(1.4)$ & $15.4(1.7)$ & $6.7(1.8)$ & $3.9(0.3)$ \\
\hline \multicolumn{7}{|l|}{ Categories, \% } \\
\hline$<15$ days & 94.9 & 84.0 & 63.2 & 43.0 & 19.8 & 81.7 \\
\hline$>=15$ and $<=30$ & 4.9 & 15.9 & 36.8 & 55.3 & 6.4 & 11.1 \\
\hline Missing & 0.2 & 0.1 & 0.0 & 1.7 & 73.8 & 7.2 \\
\hline Seen mental health professional past 12 months, $\%$ & 9.1 & 7.4 & 12.6 & 14.0 & 8.4 & 9.2 \\
\hline Antidepressants, \% & 14.1 & 24.9 & 42.2 & 49.4 & 18.1 & 19.6 \\
\hline SSRI, \% & 8.9 & 15.6 & 24.2 & 31.5 & 11.2 & 12.2 \\
\hline SSNRI, \% & 2.1 & 2.8 & 10.7 & 9.0 & 4.7 & 3.3 \\
\hline Duloxetine, \% & 1.0 & 0.3 & 1.1 & 0.0 & 0.9 & 0.8 \\
\hline TCA, \% & 2.3 & 5.5 & 8.1 & 10.1 & 1.2 & 3.4 \\
\hline Miscellaneous, \% & 3.5 & 3.3 & 8.1 & 11.7 & 4.1 & 4.1 \\
\hline -OPD, \% & 7.6 & 5.8 & 7.0 & 6.8 & 4.2 & 6.9 \\
\hline
\end{tabular}

SSRI selective serotonin reuptake inhibitors, SSNRI serotonin-norepinephrine reuptake inhibitors, TCA tricyclic antidepressants, Miscellaneous antidepressants include bupropion, vilazodone, maprotiline, mirtazapine, nefazodone and trazodone. -OPD: excluding antidepressants that are also approved for other psychiatric disorders: SSRIs escitalopram, fluoxetine, fluvoxamine, paroxetine, sertraline, and SSNRI venlafaxine 


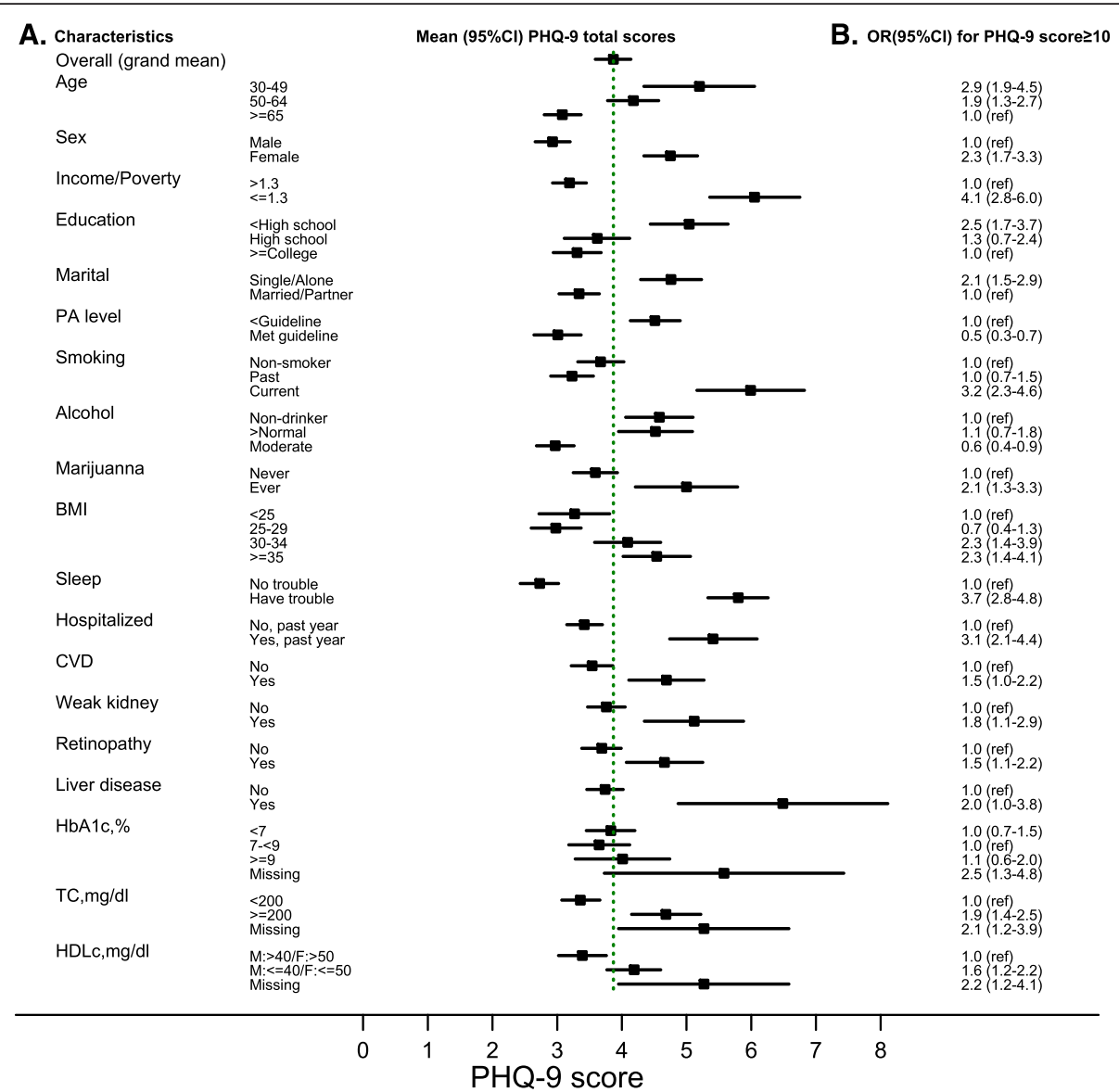

Fig. 2 Mean (95\% Cl) of PHQ-9 score (panel a) and crude ORs for CRD (panel b). Legends: squares mark out mean estimates while horizontal lines spread $95 \%$ confidence interval, the green vertical dotted-line indicates overall grand mean of PHQ-9 score (as a continuous variable) for all T2DM, not stratified by any specific characteristics. Panel a shows the mean ( $95 \% \mathrm{Cl})$ of continuous PHQ-9 score according to various characteristics in T2DM, Panel b shows corresponding crude odds ratio (OR) for clinically relevant depression (CRD), defined by PHQ-9 $\geq 10$. CVD, cardiovascular diseases; HDLC, high-density lipoprotein cholesterol; TC, total cholesterol. BMI, body mass index; PA, physical activity. While PHQ-9 score ranges from 0 to $27, x$-axis is not drawn to full 27 points to save space

We also evaluated uni-variable associations with CSD (i.e., PHQ-9 score $\geq 15$ ); variables that achieved statistical significance were a subset of those identified for CRD above. The mean PHQ-9 scores and the OR estimates for these variables were also plotted (Additional file 4) and overall, the OR estimates for the variables in association with CSD appeared larger than the corresponding estimates for CRD.

Table 4 presents results of multi-variable regression models that only retained statistically significant predictors for CRD and CSD. Significant predictors of CRD include age, gender, income, education, smoking, body mass index, sleep problems, hospitalization in the past 12 months, and total cholesterol not at goal ( $\geq 200 \mathrm{mg} /$ dl). Significant predictors of CSD include age, gender, income, education, physical activity, sleep problems, cardiovascular diseases, and total cholesterol not at goal ( $\geq 200 \mathrm{mg} / \mathrm{dl}$ ).

\section{Discussion}

To the best of our knowledge, this study is the first to provide a US national level estimates of depression prevalence for people with T2DM $[8,9]$.

We estimated an overall point prevalence of $10.6 \%$ (95 \% CI 8.9-12.2) for clinically relevant depression (CRD, PHQ-9 score $\geq 10$ ) in people with T2DM, which is about 1.56 times that of the general US adult population (6.8 \% using NHANES data 2005-2008) [27]. Similarly, our overall prevalence estimate of $4.2 \%$ for clinically significant depression (CSD, PHQ-9 score $\geq 15$ ) in people with T2DM is about 1.75 times that of the general US adult population $(2.4 \%)$ [27]. Interestingly, our study found that $49.4 \%$ of the T2DM people with CSD reported taking antidepressants, which is about 1.55 times of the $31.8 \%$ reported for the general US adult population with CSD [27]. This suggests that people with T2DM who had CSD are more likely to receive antidepressant medications 
Table 4 ORs (95\% Cls) of statistically significant predictors for CRD and CSD in the multivariable model

\begin{tabular}{|c|c|c|c|}
\hline Variable/characteristic & Category & $\begin{array}{l}\text { CRD (PHQ-9 depression } \\
\text { total scores } \geq 10 \text { ) }\end{array}$ & $\begin{array}{l}\text { CSD (PHQ-9 depression } \\
\text { total scores } \geq 15 \text { ) }\end{array}$ \\
\hline \multirow[t]{3}{*}{ Age, years } & $30-49$ & $2.0(1.3-3.3)$ & $3.3(1.5-7.0)$ \\
\hline & $50-64$ & $1.5(1.0-2.2)$ & $3.3(2.0-5.5)$ \\
\hline & $\geq 65$ & 1.0 (ref.) & 1.0 (ref.) \\
\hline \multirow[t]{2}{*}{ Sex } & Male & 1.0 (ref.) & 1.0 (ref.) \\
\hline & Female & $2.1(1.4-3.1)$ & $1.8(1.0-2.9)$ \\
\hline \multirow[t]{2}{*}{ Family income/poverty ratio } & $>1.3$ & 1.0 (ref.) & 1.0 (ref.) \\
\hline & $\leq 1.3$ & $2.8(1.8-4.4)$ & $3.1(1.6-5.8)$ \\
\hline \multirow[t]{3}{*}{ Education level } & Below high school & $2.0(1.3-3.1)$ & $3.1(1.7-5.6)$ \\
\hline & High school graduate & $1.3(0.7-2.4)$ & $1.5(0.7-3.0)$ \\
\hline & College or above & 1.0 (ref.) & 1.0 (ref.) \\
\hline \multirow[t]{2}{*}{ Physical Activity level ${ }^{a}$} & Below guideline & - & 1.0 (ref.) \\
\hline & Met guideline & - & $0.4(0.2-0.7)$ \\
\hline \multirow[t]{3}{*}{ Smoking status } & Non-smoker & 1.0 (ref.) & 1.0 (ref.) \\
\hline & Former smoker & $1.3(0.9-2.0)$ & - \\
\hline & Current smoker & $2.4(1.7-3.6)$ & - \\
\hline \multirow[t]{4}{*}{ Body Mass Index, kg/m² } & $<25$ & 1.0 (ref.) & - \\
\hline & $25-29$ & $0.9(0.4-1.8)$ & - \\
\hline & $30-34$ & $3.0(1.6-5.5)$ & - \\
\hline & $\geq 35$ & $2.4(1.3-4.6)$ & - \\
\hline \multirow[t]{2}{*}{ Sleeping status } & No trouble & 1.0 (ref.) & 1.0 (ref.) \\
\hline & Have trouble & $3.0(2.1-4.2)$ & $3.7(2.4-5.7)$ \\
\hline \multirow[t]{2}{*}{ Hospitalized in the past 12 months } & No & 1.0 (ref.) & - \\
\hline & Yes & $2.6(1.8-3.7)$ & - \\
\hline \multirow[t]{2}{*}{ Cardiovascular diseases } & No & - & 1.0 (ref.) \\
\hline & Yes & - & $2.2(1.3-3.8)$ \\
\hline \multirow[t]{2}{*}{ Total cholesterol, mg/dL } & $<200$ & 1.0 (ref.) & 1.0 (ref.) \\
\hline & $\geq 200$ & $1.7(1.2-2.4)$ & $2.0(1.2-3.6)$ \\
\hline
\end{tabular}

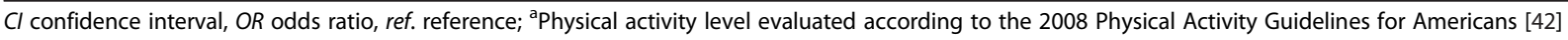

than in the general population of the US with CSD. This could be due to the higher rate of interaction of subjects with T2DM with health care providers. Likewise, when we consider antidepressant medication in combination with CRD, the burden of depressive symptoms on people with T2DM overall may be about $25.4 \%$, and in women it may be as high as about $32 \%$ (Fig. 1).

A previous meta-analysis based on studies before 2005 has reported depression prevalence of $17.6 \%$ among people with T2DM, which appeared higher than our estimated prevalence for CRD and CSD, but lower than our estimate of the burden of depression combined with antidepressant treatment. This may suggest that recognition and treatment of depression has improved for people with T2DM, even though some people may be taking the antidepressant medications for symptoms or diseases other than depression, or perhaps physicians may prescribe more antidepressants to people with chronic diseases. For example, duloxetine is a selective serotonin and norepinephrine reuptake inhibitor (SNRI) antidepressant that is also indicated for neuropathic pain for people with T2DM. However, only $0.8 \%$ of people with T2DM reported taking duloxetine (Table 3). In a sensitivity analysis that excluded antidepressants approved for other psychiatric disorders (mainly anxiety and related disorders, see Table 3, last row), our estimated overall prevalence of CRD and/or antidepressants was $16.7 \%$ (95 \% CI 14.6-18.9 \%) in people with T2DM. These estimates are likely too conservative (i.e., underestimation of the prevalent burden of depression among people with T2DM) because of (1) the assumption that none of the excluded common antidepressants were taken for depressive symptoms, or none affected PHQ-9 scores at all, and (2) the common comorbidity of depression and other psychiatric conditions, particularly anxiety disorders. For example, one Canadian community-based study of about 2,000 people with T2DM [28] found $74 \%$ 
(135 of 183) of people with above-threshold anxiety symptoms (defined as score $\geq 10$ on the 7-item Generalized Anxiety Disorder Questionnaire (GAD-7)) also had comorbid depression symptoms (defined as PHQ-9 score $\geq 10$ ). We conducted extensive literature search but couldn't find data on the prevalence of comorbid depression and anxiety among people with diabetes in the US, consistent with the observation made by the Canadian study that "despite this frequently observed co-morbidity, there is little research that explicitly looks at both anxiety and depression in people with diabetes." Even in the general US population, we only found one publication that provided data (in their Supplemental Table S3) for estimating the prevalence of co-morbid depression among people with anxiety [29]: among participants in the Detroit Neighborhood Health Study, 2008-2010, $78.9 \%$ of those with anxiety (defined as GAD-7 score 15-21) also had depression (defined as modified PHQ-9 with cutoff 3+). The prevalence of (self-reported physician diagnosed) diabetes was $18.3 \%$ among a total of 1,050 participants in that study. Furthermore, the prevalence of PHQ-9 score $\geq 10$, and/or having seen a mental health professional in the past 12 months, and/or taking antidepressants (excluding the 6 antidepressants with multiple indications) was $22.7 \%$ (95 \% CI 20.0-25.4\%) (Additional file 3). Additional analyses showed consistent positive correlation between taking antidepressants and higher PHQ-9 scores (Additional file 5).

Depression by itself has been associated with reduced quality of life, loss of work days, damage to relationships, and even suicide [30,31]. Our study has found such negative impact to be particularly concentrated among people with moderately severe or severe depressive symptoms (PHQ-9 score $\geq 15$ ), and antidepressant treatment alone may not have achieved remission of depressive symptoms for them [32]. Although NHANES is a large, nationally representative survey that provides comprehensive data, the cross-sectional data does not provide longitudinal follow-up, and effectiveness of depression medications cannot be evaluated.

Cross-sectional studies cannot clarify whether patients are depressed because they have disabling and worrisome diabetes complications, or whether having depression could actually precede occurrence of diabetes complications [33]. The biological mechanisms by which depression and type 2 diabetes are associated remain unclear [34]. In addition to a growing body of literature indicating a bidirectional association between these two serious long-term diseases [35], shared biological and behavioral pathways that may simultaneously predispose to both disorders have been proposed [36].

Depression prevalence estimates may also depend on the clinical and methodological settings, such as clinical versus community, self-reported questionnaires versus standardized diagnostic interviews, etc. [8]. Used most often in primary care settings, the PHQ-9 has been shown to be a reliable and valid tool for screening depression [37], including in the elderly [38], and has been applied in studies of diabetes [39]. In fact, some primary care practices were able to integrate PHQ-9 questionnaire with electronic health records (EHR), and such enriched medical records have helped provide higher quality care for depression [40]. Even sub-clinical depression is associated with worse self-care behavior, and may affect all aspects of diabetes treatment [5]. We have identified some easily measured significant predictors for CRD and CSD, which may help with targeted depression screening. Results of stratified analysis by common comorbidities in T2DM (regardless of statistical significance) are given in additional file 6 . The management of recognized depression in people with T2DM mainly involves psychotherapy, antidepressant medications, and collaborative care that commonly employs treatment steps or algorithms that include both some psychotherapy and antidepressant medications [41].

Missing data must also be considered in the prevalence estimates. For example, if people with severe depression are less likely to complete the PHQ-9 questionnaire, it could lead to underestimation of the prevalence of severe depression symptoms. We examined the missing data pattern, including individual questions on PHQ-9 questionnaire, but did not detect any notable patterns in demographic and general characteristics between people with missing PHQ-9 data and people with complete data. The most common pattern of missing data is missing all 9 questions.

\section{Conclusions}

The prevalence of clinically relevant depression (CRD) and clinically significant depression (CSD) among people with T2DM in the US may be lower than in earlier studies, however, the burden of depression remains high. Psychological consultation is less than $15 \%$ (Table 3) even for people with clinically significant depression. All health care providers should be aware of mental-health comorbidities while treating a the physical condition of patients with T2DM. Significant predictors of both CRD and CSD include younger age, female sex, lower education level, lower family income, and trouble sleeping. Specific predictors of CRD also include smoking, body mass index $\geq 30 \mathrm{~kg} / \mathrm{m}^{2}$, and hospitalization in the past 12 months; specific predictors of CSD included physical activity below recommended level, cardiovascular diseases, and high total cholesterol.

Further research with longitudinal follow-up for depression in people with T2DM is needed to understand real world effectiveness of depression management.

\section{Ethics approval and consent to participate}

The present study was exempt of ethics approval as a secondary analysis of existing NHANES pubic data under the 
US Health \& Human Services (HHS)' regulations at 45 CFR 46.101(b)(4) (available from http://www.hhs.gov/ohrp/ policy/cdebiol.html, last accessed December 2014). US National Center for Healthcare Statistics Research Ethics Review Board (ERB) approved the NHANES surveys (http:// www.cdc.gov/nchs/nhanes/irba98.htm, last accessed March 15, 2016). Adult participants gave written informed consent both before home interview and before the exams. Consent forms are available from http://www.cdc.gov/ nchs/nhanes.htm.

\section{Consent for publication \\ Not applicable.}

\section{Availability of data and materials}

All NHANES public-use survey data and related documents are freely available from http://www.cdc.gov/nchs/ nhanes/nhanes_questionnaires.htm, with additional weblinks provided for each 2-year survey cycle. Additional files $1,2,3,4,5$ and 6 mentioned in the main text are available as Supplemental Materials.

\section{Additional files}

Additional file 1: Title "Response rate in NHANES", provides survey response rate by each NHANES survey cycle. (DOCX $28 \mathrm{~kb}$ )

Additional file 2: Title "Characteristics of People With T2DM by PHQ-9 Depression Scores, NHANES 2005-2012", description according to 4 categories of PHQ-9 score. (DOCX $40 \mathrm{~kb}$ )

Additional file 3: Title "PHQ-9 Score and Depression Prevalence Estimates in T2DM, by NHANES survey cycles", analysis by each survey cycle. (DOCX $30 \mathrm{~kb}$ )

Additional file 4: Title "Mean (95\% Cl) of PHQ-9 score (panel A) and crude ORs for CSD (panel B).", results for CSD. (DOCX $37 \mathrm{~kb}$ )

Additional file 5: Title "Odds ratio (95\% confidence interval) for taking antidepressants (yes vs no) comparing different PHQ-9 score cutoffs lamong people with T2DM", analysis of positive correlation between PHQ-9 score and antidepressants. (DOCX $28 \mathrm{~kb}$ )

Additional file 6: Title "Stratified analysis according to comorbidities", analysis by comorbidities regardless of statistical significance. (DOCX 29 kb)

\section{Abbreviations}

BMI: body mass index; CRD: clinically relevant depression; CSD: clinically significant depression; CVD: cardiovascular diseases; HDLc: high-density lipoprotein cholesterol; MEC: mobile examination center; NHANES: the National Health and Nutrition Survey; PA: physical activity; PHQ-9: Patient Health Questionnaire, 9; TC: total cholesterol; US: United States; T2DM: type 2 diabetes mellitus.

\section{Competing interests}

YW, JMSL, SCB and PES are employed, and VJZ used to be employed by Janssen Pharmaceutical Companies of Johnson\&Johnson.

\section{Authors' contributions}

YW and PES participated in the conception and design of the study; JMSL and SCB coordinated the study and design; VJZ and YW performed the analyses; YW and PES wrote the first draft of the manuscript. All authors participated in the writing and revision of the successive drafts of the manuscript, interpretation of the results, and approved the final study design and manuscript.

\section{Acknowledgements}

The authors thank the NHANES participants and the staff numbers for their contributions of the data and data collection. The authors thank Marcia FT Rupnow for her input into the study design, and Gayle Murray for her help in the publication process.

\section{Funding}

None.

\section{Author details}

'Janssen Research \& Development, LLC, 1125 Trenton Harbourton Road, Titusville, NJ 08560, USA. ${ }^{2}$ Janssen Scientific Affairs, LLC, Raritan, 1000 US Route 202, Raritan, NJ 08869, USA. ${ }^{3}$ Department of Public Health Sciences, Medical University of South Carolina, 135 Cannon Street Suite 303, Charleston, SC 29425, USA.

Received: 10 September 2015 Accepted: 31 March 2016 Published online: 05 April 2016

\section{References}

1. Lustman PJ, Anderson RJ, Freedland KE, de Groot M, Carney RM, Clouse RE. Depression and poor glycemic control: a meta-analytic review of the literature. Diabetes Care. 2000:23(7):934-42.

2. Bogner HR, Morales KH, de Vries HF, Cappola AR. Integrated management of type 2 diabetes mellitus and depression treatment to improve medication adherence: a randomized controlled trial. Ann Fam Med. 2012; 10(1):15-22.

3. Atlantis E, Fahey P, Foster J. Collaborative care for comorbid depression and diabetes: a systematic review and meta-analysis. BMJ Open. 2014;4(4): e004706.

4. American Association of Clinical Endocrinologists. Management of common comorbidities of diabetes [http://outpatient.aace.com/type-2diabetes/management-of-common-comorbidities-of-diabetes, last accessed July 2015]

5. Hermanns N, Caputo S, Dzida G, Khunti K, Meneghini LF, Snoek F. Screening, evaluation and management of depression in people with diabetes in primary care. Prim Care Diabetes. 2013;7(1):1-10.

6. Ducat $L$, Philipson $L H$, Anderson BJ. Routine depression screening for patients with diabetes-reply. Jama. 2014;312(22):2413.

7. Eaton WW. Epidemiologic evidence on the comorbidity of depression and diabetes. J Psychosom Res. 2002;53(4):903-6.

8. Anderson RJ, Freedland KE, Clouse RE, Lustman PJ. The prevalence of comorbid depression in adults with diabetes: a meta-analysis. Diabetes Care. 2001;24(6):1069-78.

9. Ali S, Stone MA, Peters JL, Davies MJ, Khunti K. The prevalence of co-morbid depression in adults with type 2 diabetes: a systematic review and metaanalysis. Diabet Med. 2006:23(11):1165-73.

10. Nichols GA, Brown JB. Unadjusted and adjusted prevalence of diagnosed depression in type 2 diabetes. Diabetes Care. 2003;26(3):744-9.

11. Li C, Ford ES, Strine TW, Mokdad AH. Prevalence of depression among U.S. adults with diabetes: findings from the 2006 behavioral risk factor surveillance system. Diabetes Care. 2008:31(1):105-7.

12. About the National Health and Nutrition Examination Survey [http://www. cdc.gov/nchs/nhanes/about_nhanes.htm, last accessed 15 Mar 2016]

13. Okosun IS, Annor FB, Seale JP, Eriksen MP. Abdominal adiposity and family income-to-poverty ratio in American women. Obes Res Clin Pract. 2014; 8(3):e201-98.

14. Cerner Multum. Lexicon [http://www.multum.com/Lexicon.htmlllast accessed 15 Mar 2016]

15. Mezuk B, Johnson-Lawrence V, Lee H, Rafferty JA, Abdou CM, Uzogara EE, Jackson JS. Is ignorance bliss? Depression, antidepressants, and the diagnosis of prediabetes and type 2 diabetes. Health Psychol. 2013;32(3): 254-63.

16. Kroenke K, Spitzer R. The PHQ-9: A new depression diagnostic and severity measure. Psychiatr Ann. 2002;32(9):509-15.

17. Kroenke K, Spitzer RL, Williams JB. The PHQ-9: validity of a brief depression severity measure. J Gen Intern Med. 2001;16(9):606-13.

18. Manea L, Gilbody S, McMillan D. Optimal cut-off score for diagnosing depression with the Patient Health Questionnaire (PHQ-9): a meta-analysis. CMAJ. 2012;184(3):E191-6. 
19. Agency for Healthcare Research and Quality. Depression: percent of clinically significant depression patients who attain a 5 point or greater reduction in Patient Health Questionnaire (PHQ) score within 6 months after their New Episode PHQ [http://www.qualitymeasures.ahrq.gov/content. aspx?id=27605, last accessed 15 Mar 2016]

20. Uebelacker LA, German NM, Gaudiano BA, Miller IW: Patient health questionnaire depression scale as a suicide screening instrument in depressed primary care patients: a cross-sectional study. Prim Care Companion CNS Disord 2011, 13(1) doi: 10.4088/PCC.10m01027.

21. Dodd AH, Colby MS, Boye KS, Fahlman C, Kim S, Briefel RR. Treatment approach and HbA1c control among US adults with type 2 diabetes: NHANES 1999-2004. Curr Med Res Opin. 2009;25(7):1605-13.

22. Reinstatler L, Qi YP, Williamson RS, Garn JV, Oakley Jr GP. Association of biochemical $B(1)(2)$ deficiency with metformin therapy and vitamin $B(1)(2)$ supplements: the National Health and Nutrition Examination Survey, 1999-2006. Diabetes Care. 2012;35(2):327-33.

23. Roy T, Lloyd CE. Epidemiology of depression and diabetes: a systematic review. J Affect Disord. 2012;142(Suppl):S8-21.

24. Henderson HV, Velleman PF. Building multiple regression models interactively. Biometrics. 1981;37:391-411.

25. Tibshirani R. Regression shrinkage and selection via the lasso. J R Stat Soc Series B. 1996;58:267-88.

26. Altman DG, Andersen PK. Bootstrap investigation of the stability of a Cox regression model. Stat Med. 1989;8(7):771-83.

27. Shim RS, Baltrus P, Ye J, Rust G. Prevalence, treatment, and control of depressive symptoms in the United States: results from the National Health and Nutrition Examination Survey (NHANES), 2005-2008. J Am Board Fam Med $2011 ; 24(1): 33-8$

28. Smith KJ, Schmitz N. Association of depression and anxiety symptoms with functional disability and disability days in a community sample with type 2 diabetes. Psychosomatics. 2014;55(6):659-67.

29. Demmer RT, Gelb S, Suglia SF, Keyes KM, Aiello AE, Colombo PC, Galea S, Uddin M, Koenen KC, Kubzansky LD. Sex differences in the association between depression, anxiety, and type 2 diabetes mellitus. Psychosom Med. 2015;77(4):467-77.

30. Bernal M, Haro JM, Bernert S, Brugha T, de Graaf R, Bruffaerts R, Lepine JP, de Girolamo G, Vilagut G, Gasquet I, et al. Risk factors for suicidality in Europe: results from the ESEMED study. J Affect Disord. 2007;101(1-3):27-34.

31. Stewart WF, Ricci JA, Chee E, Hahn SR, Morganstein D. Cost of lost productive work time among US workers with depression. Jama. 2003; 289(23):3135-44

32. Mauskopf JA, Simon GE, Kalsekar A, Nimsch C, Dunayevich E, Cameron A. Nonresponse, partial response, and failure to achieve remission: humanistic and cost burden in major depressive disorder. Depress Anxiety. 2009;26(1): 83-97.

33. Lin EH, Rutter CM, Katon W, Heckbert SR, Ciechanowski P, Oliver MM, Ludman EJ, Young BA, Williams LH, McCulloch DK, et al. Depression and advanced complications of diabetes: a prospective cohort study. Diabetes Care. 2010;33(2):264-9.

34. Berge LI, Riise T. Comorbidity between type 2 diabetes and depression in the adult population: directions of the association and its possible pathophysiological mechanisms. Int J Endocrinol. 2015;2015:164760.

35. Golden SH, Lazo M, Carnethon M, Bertoni AG, Schreiner PJ, Diez Roux AV, Lee HB, Lyketsos C. Examining a bidirectional association between depressive symptoms and diabetes. Jama. 2008:299(23):2751-9.

36. Holt Rl, de Groot M, Lucki I, Hunter CM, Sartorius N, Golden SH. NIDDK international conference report on diabetes and depression: current understanding and future directions. Diabetes Care. 2014;37(8):2067-77.

37. Arroll B, Goodyear-Smith F, Crengle S, Gunn J, Kerse N, Fishman T, Falloon K, Hatcher S. Validation of PHQ-2 and PHQ-9 to screen for major depression in the primary care population. Ann Fam Med. 2010;8(4):348-53.

38. Phelan E, Williams B, Meeker K, Bonn K, Frederick J, Logerfo J, Snowden M. A study of the diagnostic accuracy of the $\mathrm{PHQ}-9$ in primary care elderly. BMC Fam Pract. 2010;11:63.

39. Katon W, von Korff M, Ciechanowski P, Russo J, Lin E, Simon G, Ludman E, Walker E, Bush T, Young B. Behavioral and clinical factors associated with depression among individuals with diabetes. Diabetes Care. 2004;27(4):914-20.

40. Valuck RJ, Anderson HO, Libby AM, Brandt E, Bryan C, Allen RR, Staton EW, West DR, Pace WD. Enhancing electronic health record measurement of depression severity and suicide ideation: a Distributed Ambulatory Research in Therapeutics Network (DARTNet) study. J Am Board Fam Med. 2012;25(5): 582-93.

41. Markowitz SM, Gonzalez JS, Wilkinson JL, Safren SA. A review of treating depression in diabetes: emerging findings. Psychosomatics. 2011;52(1):1-18.

42. US Department of Health and Human Services (HHS): 2008 Physical Activity Guidelines for Americans. [http://www.health.gov/paguidelines/pdf/paguide. pdf, last accessed 15 Mar 2016]

\section{Submit your next manuscript to BioMed Central and we will help you at every step:}

- We accept pre-submission inquiries

- Our selector tool helps you to find the most relevant journal

- We provide round the clock customer support

- Convenient online submission

- Thorough peer review

- Inclusion in PubMed and all major indexing services

- Maximum visibility for your research

Submit your manuscript at www.biomedcentral.com/submit
Biomed Central 